\title{
Coulisses
}

Revue de théâtre

41 | Automne 2010

Le dialogue orient-occident

\section{Deux pièces Nô}

Présentation

\section{Coulisses}

\section{OpenEdition}

\section{Journals}

Édition électronique

URL : https://journals.openedition.org/coulisses/1439

DOI : 10.4000/coulisses. 1439

ISSN : 2546-9460

Éditeur

Presses universitaires de Franche-Comté

\section{Édition imprimée}

Date de publication : 31 décembre 2010

Pagination : 77-80

ISBN : 978-2-84867-302-8

ISSN : $1150-594 \mathrm{X}$

\section{Référence électronique}

Coulisses, «Deux pièces Nô », Coulisses [En ligne], 41 | Automne 2010, mis en ligne le 30 novembre 2016, consulté le 30 décembre 2022. URL : http://journals.openedition.org/coulisses/1439; DOI : https://doi.org/10.4000/coulisses. 1439 


\title{
Deux pièces Nô
}

\author{
Présentation
}

Coulisses

\section{RÉFÉRENCE}

\section{Zeami, Kiyotsune}

Jannette Cheong, Pagode

Direction artistique : Richard Emmert. Le Théâtre Nô Oshima et le Théâtre Nohgaku.

Théâtre Nô Classique et Contemporain, Londres-Dublin-Oxford-Paris, décembre 2009.

1 En 2009, début décembre, Le Théâtre Nô Oshima et le Théâtre Nohgaku ont présenté à Londres (2-3, Southbank Centre), Dublin (5, Samuel Beckett Theatre), Oxford (7, O'Reilly Theatre) et à Paris (9-10, Maison du Japon), deux pièces de Nô, l'une classique Kiyotsune, l'autre contemporaine Pagode.

2 Le Théâtre Nô Oshima tire sa légitimité de l'École Kita, l'une des cinq troupes originelles du Nô. Masanobu Oshima, chef de troupe, a été lui-même élève de Kita Minoru, quinzième maître de l'École Kita. Il est dans Kiyotsune le « shite », c'est-à-dire le protagoniste. Kinue Oshima, la fille de Masanobu, joue également le rôle de «shite " dans Pagode. C'est la première femme que l'École Kita a consacrée comme acteur de Nô (1998). Teruhisa Oshima, fils de Masanobu, shite de l'Acte II dans Pagode, a été formé lui aussi à l'École Kita. Masanobu, Kinue et Teruhisa se sont produits sur beaucoup de scènes de Nô au Japon comme à l'étranger.

Kinue Oshima explique à Nicole Salez dans tout pour les femmes.com (9:12:09) ce qui par rapport au chant la différencie d'un acteur : «La tonalité de la voix de la femme peut entraîner un désaccord par rapport à celle des voix d'homme. C'est pourquoi, il m'est assez rare de participer aux chœurs. Je joue souvent des rôles qui n'ont que des chants solo. Quand le rôle me demande des chants qui répondent au chœur, il m'est plus 
simple de collaborer avec des membres du chœur avec lesquels j'ai l'habitude de travailler car ils s'adaptent mieux à ma voix. Ceci étant, on me dit que le chant de nô ne dépend pas du timbre de la voix mais de la profondeur du souffle. C'est pour moi un aspect à approfondir. » Parlant des costumes, elle ajoute : «Comme très peu sont faits sur mesure pour les acteurs (trop chers à refaire), et comme je suis assez menue, il arrive que le costume soit trop grand pour moi. Je suis obligée de faire avec. Une petite information supplémentaire, les costumes anciens sont, pour moi, plus faciles à porter. Il semblerait que les Japonais d'autrefois étaient plus petits!» Au sujet de Pagode, dont elle a créé la chorégraphie, et dont elle est l'actrice principale (le shite), elle sourit en avouant qu'elle $\mathrm{a}$ eu du mal à prononcer correctement certains mots anglais !

米

4 Le Théâtre Nohgaku est une compagnie internationale composée de membres japonais et nord-américains : sa raison d'être est de créer et de présenter des pièces de Nô en anglais dans le respect des règles traditionnelles de cette forme de théâtre (scène, musique, costumes, masques). Le fondateur et directeur du Théâtre Nohgaku, Richard Emmert, est lui aussi diplômé de l'École Kita. Depuis 1973, il étudie, enseigne le Nô et interprète des pièces du répertoire à divers titres. Il a donné de nombreuses conférences, fait des démonstrations et animé des ateliers au Japon, aux États-Unis, en Europe, en Australie, en Chine (Hong Kong). Au Japon et aux États-Unis en été, il dirige le Noh Training Project. Il a composé et arrangé la musique de plusieurs pièces de Nô en anglais. Enfin il est le co-auteur de sept guides de spectacles de Nô publiés par le Théâtre National de Nô et il enseigne le théâtre et la musique asiatiques à l'Université Musashino de Tokyo. Dans une interview donnée en janvier 2010 à La Terrasse (propos recueillis et traduits par Agnès Santi), Richard Emmert revient sur ses débuts : "Au Japon, quelqu'un m'a demandé dans les années 80 de travailler sur Au puits de l'épervier de William Butler Yeats, influencé par le style nô. Puis comme je ne voulais pas de comédiens de style occidental mais vraiment des acteurs nô, j'ai constitué un atelier permanent en 1991 à Tokyo pour des étrangers, puis en 95 un atelier d'été intensif aux États-Unis. Nous avons étudié la danse, le chant avant d'introduire l'étude d'instruments...»

\section{米}

5 La représentation à la maison de la Culture du Japon a été, sur le plan du spectacle, magnifique (costumes, chants, danse), mais comme le texte de la pièce n'était pas entre toutes les mains et qu'il n'y avait pas de surtitrage, à la fois le sens et la beauté du texte n'ont pu être saisis. Richard Emmert (qui jouait le rôle de l'épouse de Kiyotsune) met au premier rang des pièces de guerriers Kiyotsune, avec Atsumori, parce qu'elle représente bien à ses yeux la lassitude des combats et qu'à ce titre elle pouvait être comme une catharsis pour des samouraï, qu'elle pouvait libérer d'affects longtemps refoulés dans le subconscient et responsables de traumatismes psychiques. De plus, le dialogue entre le guerrier Kiyotsune et son épouse, marqué par des échanges d'une rare intensité, étonne dans le Nô par la force d'expression accordée à une femme qui parle d'égal à égal à son compagnon et qui lui reproche un suicide qui la prive de sa présence et de son amour. 
Pagode (une pièce nô en anglais), qui a été présentée en deuxième partie de spectacle à la Maison du Japon, est le fruit d'une collaboration étroite entre Richard Emmert et l'auteure Jannette Cheong.

Pour rendre compte de cette entreprise commune, le mieux est de reproduire intégralement ici les propos recueillis par Agnès Santi pour La Terrasse, janvier 2010, $\mathrm{n}^{\circ} 74$.

Entretien avec Jannette Cheong et Richard Emmert, La Terrasse, janvier 2010, $\mathrm{n}^{\circ} 74$, propos recueillis par Agnès Santi

http://www.journal-laterrasse.fr/conversation-jannette-cheong-et-richardemmert/

Agnès SANTI - Comment vous êtes-vous rencontrés ?

Jannette CHEONG - J'ai connu Richard lors d'un voyage au Japon, où j'ai aussi rencontré la famille Oshima, une famille nô traditionnelle possédant son propre théâtre. J’ai été fascinée par leur art et leur discipline d'acteur. Lorsque je leur ai raconté l'histoire de la pièce de théâtre musicale sur laquelle je travaillais, ils m'ont dit que cela ferait vraiment une bonne pièce nô ! Richard m'a enseigné les structures et les conventions du nô, et je me suis sentie très libre dans le choix des mots, des idées et des images malgré ce cadre très défini, paradoxalement libérateur.

Richard EMMERT - Nous nous sommes vraiment rencontrés en 2008 lors d'un atelier d'écriture, que nous organisons chaque année à Tokyo ou aux États-Unis. Les grandes lignes de l'histoire étaient déjà en place. Le processus nô a consisté notamment à couper, à épurer.

Agnès SANTI - En quoi cette histoire est-elle fondée sur des éléments autobiographiques ?

Jannette CHEONG - Mon père est mort subitement en 1973. Après sa mort nous avons réalisé que nous ne savions rien de lui, sauf qu'il venait de Chine. Je suis donc partie là-bas en 1975, pour découvrir où il était né, dans un pays encore très fermé même si la fin officielle de la Révolution culturelle approchait. J'enseignais l'anglais dans une zone très isolée et froide, au nord-est de la Chine. J'ai retrouvé sa trace grâce à une enveloppe avec une adresse (tout ce que j'avais!) et à un énorme livre de la bibliothèque de l'université contenant tous les noms des communes de Chine. J'ai entrepris un long voyage jusqu'à son village situé dans le sud-est. Nous avons pu rencontrer sa plus jeune sœur, qui a raconté leur enfance. Mon père vivait dans une famille très pauvre, avec ses cinq frères et sœurs, et à la fin des années 20 tous souffraient de la famine. Sa mère a pensé que, comme beaucoup d'autres enfants, il allait mourir si elle ne trouvait pas un moyen de le faire partir. Ils ont réussi à le faire engager comme mousse sur un bateau. J'ai ensuite découvert que ces bateaux transportaient du ravitaillement d'Amérique jusqu'en Angleterre pendant la seconde guerre mondiale. La pièce nô s'articule autour de la mère, dont le sacrifice a permis la survie de son fils.

Richard EMMERT - Dans les pièces nô, un voyageur se rend dans un endroit où naît une histoire avec des esprits, qui apparaissent ou peut-être sont issus des rêves du voyageur. Le père de Jannette voyage, mais le véritable voyageur, qui témoigne, c'est Jannette. La pièce nô est en cela très différente de la pièce musicale que Jannette a 
écrite, qui s'apparente à une épopée occidentale classique, centrée sur le périple du père.

Agnès SANTI - Quelles sont donc les caractéristiques spécifiques de cette pièce nô ?

Jannette CHEONG - Lorsque j'ai visité le lieu de naissance de mon père, on m'a emmenée voir une pagode, liée à une légende vieille d'un siècle, à propos d'une femme noble qui avait perdu son mari, capitaine d'un bateau naufragé. Elle n'a jamais cru en sa mort et chaque jour montait sur la colline pour le voir. Elle a commencé à construire cette pagode, chaque étage lui permettant de regarder plus loin. Lorsque le mari a pu enfin revenir, il a vu cette pagode et n'a pas reconnu sa maison, il est reparti et ils ne se sont jamais revus. J'ai voulu réunir ces deux histoires, la légende de la Pagode et l'histoire de ma grand-mère, désirant revoir son fils. Ces deux femmes courageuses partagent ce désir de revoir un être cher perdu dans des circonstances tragiques. C'est le fil commun entre ces deux histoires. Toute la pièce a lieu à côté de la Pagode. Ecrire cette pièce a constitué une expérience puissante et profonde. Cela m'a beaucoup appris sur l'identité et l'émigration, souvent une question de survie.

6 Même si Pagode était en anglais, l'absence de surtitrage, comme pour Kiyotsune, a pénalisé la pièce. Le choeur en particulier était difficilement compréhensible, même pour des anglophones. Seule parmi les acteurs, la voix du waki (la voyageuse) était parfaitement audible. C'était celle de Jubilith Moore, membre fondateur du Théâtre Nohgaku, et élève du maître de Nomura Shiro de l'École Kanze. Le public parisien a apprécié Pagode peut-être plus que Kiyotsune pour plusieurs raisons : la forte présence du waki comme je l'ai déjà relevé, un plus grand nombre de personnages, la beauté des costumes, le mystère des masques spécialement conçus pour cette pièce et les danses finales parfaitement exécutées des deux actes. Bien sûr, la résonance actuelle de Pagode, drame familial et de l'immigration forcée ne peut que nous toucher. La performance technique, la maîtrise des corps des acteurs étaient impressionnantes qu'ils soient japonais ou européens. Si les instrumentistes étaient tous japonais, par contre le chœur n'était pratiquement composé que des membres nord-américains du Théâtre Nohgaku. Richard Emmert a composé la musique de ce nô.

米

Site du Théâtre Nohgaku :

http://www.theatrenohgaku.org/

Pour voir une autre œuvre du Theatre Nohgaku «Eliza», aller sur le site: http:// www.gesticularia.fr/, entrez-y, allez à la rubrique et cliquez sur Eliza. 
INDEX

oeuvrecitee Pagode, Kiyotsune

Palavras-chave : nô, teatro japonês

Palabras claves : nô, teatro japonés

Keywords : nô, japanese theater

Mots-clés : nô, théâtre japonais 